\title{
Activation of the primary motor cortex using fully-implanted electrical sciatic nerve stimulation
}

\author{
XIAODONG LV $^{1 *}$, RONGYU TANG ${ }^{1 *}$, ZHAOLONG GAO ${ }^{2}$, DINGYIN HU ${ }^{1}$, \\ GUANGHUI LI ${ }^{1}$, YIRAN LANG ${ }^{1}$ and JIPING HE ${ }^{1}$ \\ ${ }^{1}$ Beijing Advanced Innovation Center for Intelligent Robot and System, Beijing Institute of Technology, Beijing 100081; \\ ${ }^{2}$ Neural Interface and Rehabilitation Technology Research Center, School of Automation, \\ Huazhong University of Science and Technology, Wuhan, Hubei 430074, P.R. China
}

Received March 6, 2019; Accepted July 12, 2019

DOI: $10.3892 /$ etm.2019.7993

\begin{abstract}
Functional degradation of the motor cortex usually results from brain injury, stroke, limb amputation, aging or other diseases. Currently, there are no ideal means of treatment, other than medication and sports rehabilitation. The present study investigated whether electrical stimulation of the sciatic nerve can activate the motor-related area of the brain. The study is based on a self-developed fully implantable nerve electrical stimulator and a self-developed multi-channel electroencephalogram (EEG) electrode array. The sciatic nerves of Sprague-Dawley rats (sorted into old and young groups) were stimulated by the electrical stimulator under anesthesia, and the EEG signal was recorded simultaneously. The relationship between sciatic nerve stimulation and brain activity was analyzed. The results showed that when the sciatic nerve was stimulated by the implanted electrical stimulator, motor-related channels were activated, causing contraction of the left leg. It was found that at the frequency band of $8-16 \mathrm{~Hz}$, the EEG signal in the right motor area was higher than at other frequency bands. This phenomenon was identical in both young and old rats. The results indicated that electrical stimulation of the sciatic nerve can activate the motor region of the rat brain, and provided evidence that stimulation of the sciatic nerve could be a method of preventing motor cortex degeneration.
\end{abstract}

Correspondence to: Dr Yiran Lang, Beijing Advanced Innovation Center for Intelligent Robot and System, Beijing Institute of Technology, 5 South Zhongguancun Street, Haidian, Beijing 100081, P.R. China

E-mail: yiran.lang@bit.edu.cn

${ }^{*}$ Contributed equally

Key words: implantable stimulator, electroencephalogram, electrical stimulation, stroke, wavelets

\section{Introduction}

Degeneration of the cortex is commonly found with brain injury (1), stroke (2), limb amputation (3) and aging (4). The long-term pathological state further hinders future treatments.

Hemiplegia is generally a symptom of stroke (when a bleed or blood clot damages part of brain) and brain injury, and it is generally treated with drugs $(5,6)$ such as acetyl glutamine and amantadine, physical therapy (7), general nursing methods (8), transcutaneous electrical nerve stimulation (9-12), and implanted nerve electrical stimulators (13). Many studies have shown that it is possible for patients with hemiplegia to regain motor ability in the lower limbs using certain treatments $(8,9,14,15)$. However, prolonged use of drug treatments may affect other regions of the brain, and physical care is time intensive. Neuromuscular electrical stimulation is effective in the short term in improving upper limb impairment in individuals with chronic stroke (16). Wilson et al (17) studied patients with hemiplegic shoulder pain who received a fully-implanted electrical stimulator and were followed up for 24 months; the study demonstrated the safety and efficacy of a fully-implantable axillary peripheral nerve stimulation system for chronic hemiplegic shoulder pain. In general, electrical stimulation of nerves is an efficient approach $(16,18,19)$ with minimal side effects $(17,20)$.

By implanting an electrical stimulator, studies have shown that patients with spinal cord injury can achieve standing and walking (21-23). Furthermore, research has indicated that electrical stimulation can improve cognitive deficits associated with traumatic brain injury (24), and that low-frequency electroencephalogram (EEG) signals appear when the sub-paresthesia spinal cord of a rat is stimulated (25). To date, implantable electrical stimulator microsystems have been rapidly developed and used in many fields of medicine $(13,17,26)$. However, to the best of our knowledge, there have been no studies on the effect of electrical stimulation of the sciatic nerve on the motor cortex. The present study discusses another method for the activation of motor brain regions using a peripheral nerve electrical stimulator.

The present study is based on a set of self-developed high-density electrodes for rats (27), designed to monitor EEG activity commonly used in neural interfaces $(28,29)$. 
A self-developed fully-implantable electrical stimulator was implanted subcutaneously in rats, for which the waveform amplitude, frequency and stimulation time could be set externally.

\section{Materials and methods}

Animal selection. A total of 10 healthy 8-week-old male SD rats (weight range, 250-350 g), 3 healthy 1-year-old male SD rats (weight range, 600-650 g) were selected and all rats were subjected to electrical stimulation and non-electrical stimulation. The rats were housed at $30^{\circ} \mathrm{C}$, with $55.3 \%$ humidity and a 12-h light/dark cycle, with access to food and water ad libitum. All animal experiments were performed in accordance with the Guide for the Care and Use of Laboratory Animals (30). The procedures in the study were designed to minimize the pain or discomfort of the animals, in accordance with the current protocols approved by the Laboratory Animal Ethics Committee of Beijing Institute of Technology (Beijing, China). A systematic diagram of the experiment is shown in Fig. 1.

Implantable electrical stimulator design. The present study is based on a self-developed implanted voltage stimulator and a self-developed electrode. The location of fixing of the electrodes is shown in Fig. 2A. The longitudinal spacing of the electrode points was $2 \mathrm{~mm}$, the horizontal electrode point spacing was $1.25 \mathrm{~mm}$, and the total number of electrodes was 34. A schematic representation of the implantable electrical stimulator and wave of stimulation is shown in Fig. 2C. According to function, the stimulator was divided into a power amplification module, signal processing module, low-power Bluetooth module, voltage conversion module and power supply module. The circuit hardware is shown in Fig. 2B.

Characteristics of the electrical stimulator. The amplitude, period and pulse width of the electrical stimulation waveform of the stimulator can be adjusted; there were two independently-programmable channels, so that an effective stimulation model could be set up according to different stimulation parameters. Low-power Bluetooth data transmission was based on Bluetooth 4.0 protocol data transmission, and the stimulus waveform was outputted by receiving the stimulation parameters (stimulation waveform, stimulation period and stimulation pulse width) from the host computer.

Based on the small size and low power consumption of the silicone package (when not working, the quiescent current loss was $<1 \mathrm{~mA}$, and the power consumption was $0.3 \mathrm{~W}$ when working). An LED on the chip indicated the working status. Due to its wireless charging function, there is no need to frequently remove the stimulator from the subject for charging. The stimulator supports external control terminals, including Bluetooth-enabled devices such as mobile phones and computers. In this experiment, the stimulator was controlled using an Android ${ }^{\mathrm{TM}}$ application developed based on the HMBLEComAssistant tool (http://www.huamaosoft. com/download.asp).

Surgical procedure. Firstly, SD rats were anesthetized with isoflurane and then placed on a rat stereotaxic apparatus. The pressure of the respirator was maintained at $50 \mathrm{kPa}$ and the air

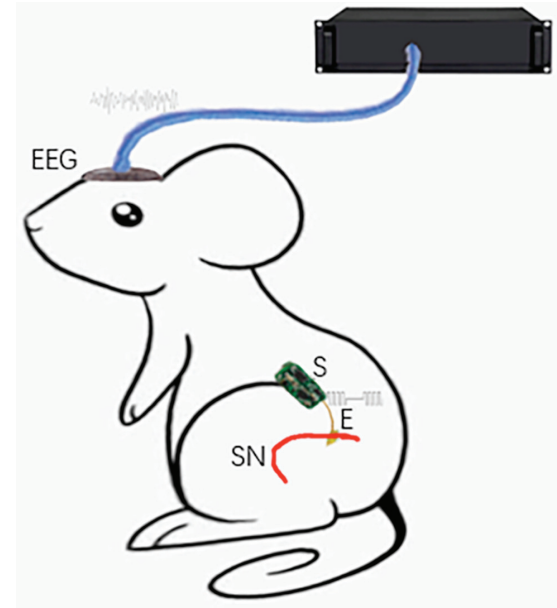

Figure 1. Systematic diagram of the experiment. S, implantable electrical stimulator; E, electrode; $\mathrm{SN}$, sciatic nerve.

flow rate was $600 \mathrm{ml} / \mathrm{min}$. The anesthetic gas concentration was $2 \%$ and a heating blanket was used to maintain normal body temperature.

The hair on the left hind leg and head of the SD rat were removed. Then, a 4-cm-long incision in the longitudinal direction was made with a scalpel on the thigh and expanded to expose the sciatic nerve. The skin and muscle of the SD rat were separated carefully using medical scissors to facilitate the implantation of the stimulator. The stimulator was implanted and the nerve of the SD rat was wrapped with the flexible electrode (shown in Fig. 2B). The incision was sutured after implantation. Next, a $5-\mathrm{cm}$ incision to the head was cut longitudinally with a scalpel. The subcutaneous tissues were removed from the skull, and the periosteum was cleaned using cotton swabs. The aim of this procedure was to make the bregma and lambda landmarks of the skull more clearly visible. The electrodes were positioned in alignment with the two markers and fixed to the skull with micro-screws (Fig. 3). After fixation of the electrode, the outlet of the electrode was covered with dental cement to separate the electrode from the fur and prevent injury caused by the movement of the rats or the insertion and removal process.

After the surgery, the rats were allowed to recover for 2-3 days and were then subjected to an electrical stimulation test. The stimulus parameters were set by amplitude and frequency. In a previous study, the square wave showed a relatively good effect on nerve stimulation (31). Therefore, the square wave waveform was selected as the output waveform of the stimulator, the carrier frequency was $1 \mathrm{kHz}$, the number of carriers was set to 2 and the stimulus voltage was set between +5 and $-5 \mathrm{~V}$. Each set of experiments took $5 \mathrm{~min}$, including 3 min of non-electrical stimulation and a 2 min of electrical stimulation. Four sets of experiment were performed every two days and the time interval between each set of experiments was $10 \mathrm{~min}$. During the process of stimulation lasting 1 month, EEG signals were acquired using a wired recording system (Cerebus ${ }^{\mathrm{TM}}$; Blackrock Microsystems LLC). After each set of experiments, a 10-min rest period was given, in order to avoid muscle fatigue, however in the ending of 1 month, one rat was paralyzed. 
A

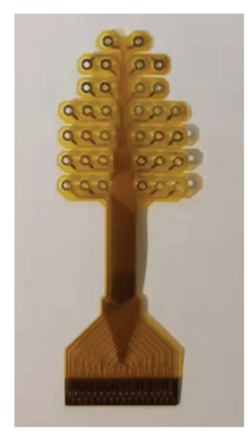

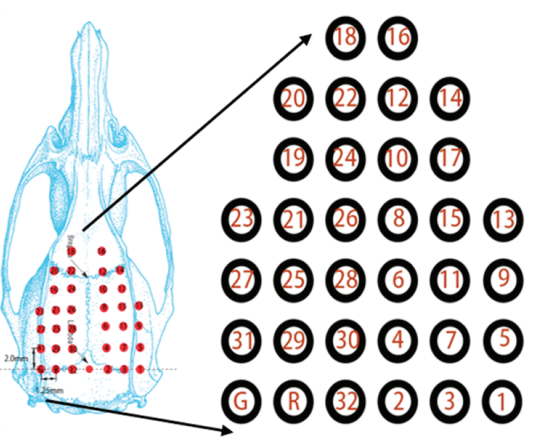

B

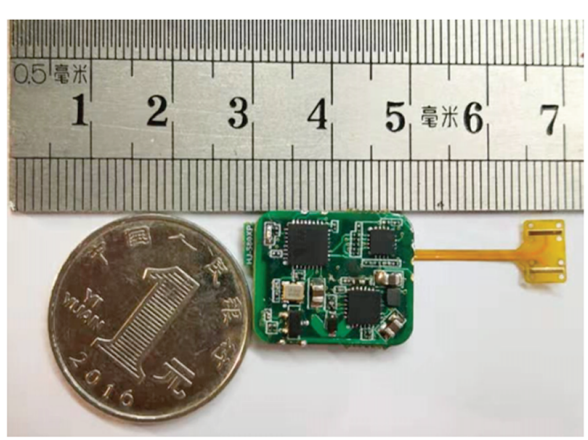

C

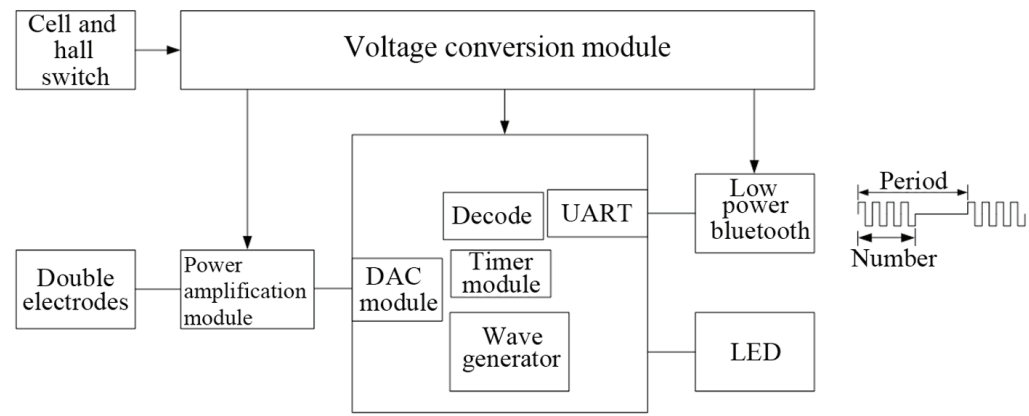

Figure 2. Self-developed devices. (A) Electroencephalography electrode array and location; (B) Photograph of the implantable electrical stimulator; (C) A schematic representation of the implantable electrical stimulator and wave of stimulation. DAC, digital-to-analog converter; UART, universal asynchronous receiver/transmitter.

Statistical analysis. Statistical analyses were carried out using EEGLAB (Swartz Center for Computational Neuroscience, University of California, San Diego, San Diego, CA, USA; https://sccn.ucsd.edu/eeglab/index.php) (32) with MATLAB 2018b (Mathworks Inc). The Wavelet Analysis v.1.0.3 (https://pypi.org/project/PyWavelets/) and SciPy v.1.3.0 (https://www.scipy.org/) expansion packages of Python v.3.7.3 (https://www.python.org/) were also used. The power map was obtained using EEGLAB by importing stored data, and Wavelet Analysis was used to analyze the relationship between voltage and frequency.

\section{Results}

Data acquisition and filtering. EEG data of the rats were acquired using a system from a wired recording system, which includes 32 signal channels with a sampling frequency of up to $1 \mathrm{kHz}$. After band-pass filtering, the signals averaged between 0.1 and $500 \mathrm{~Hz}$ using a sixth-order Chebyshev filter, and signals were notched at $50 \mathrm{~Hz}$.

Visual observation. When the sciatic nerve was stimulated, the rat leg contracted at the knee joint and extended at the ankle. In Fig. 4, the blue line represents the initial state and the red line represents the end state. Change in ankle and knee angles are shown in Fig. 4. The initial ankle and knee angles were $\sim 65^{\circ}$ and $90^{\circ}$, and $\sim 80^{\circ}$ and $78^{\circ}$ after electrical stimulation, therefore, the change in ankle and knee angles were $\sim 15^{\circ}$ and $12^{\circ}$, respectively. The angle of ankle and knee angles have changed markedly before and after electrical stimulation.

Power map. As shown in Fig. 5A and B, the EEG power of the younger rats in the non-stimulation group was lower than that

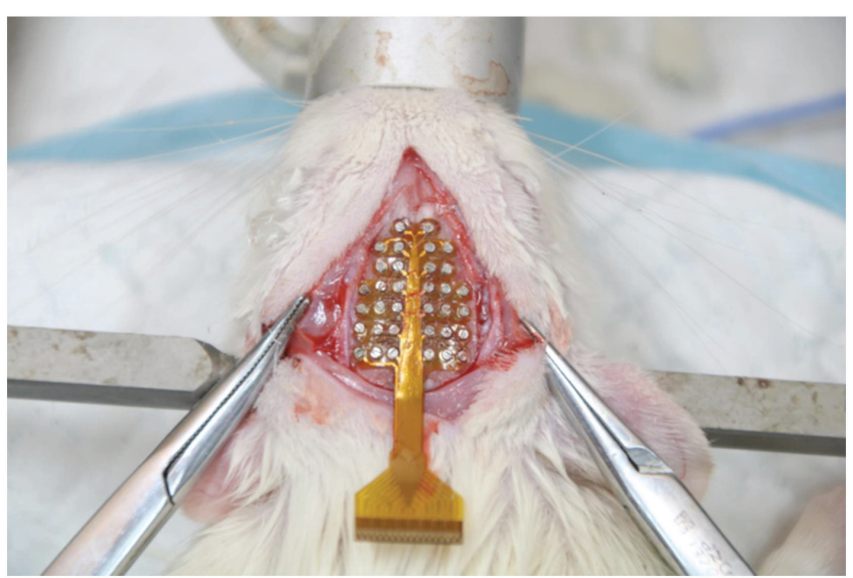

Figure 3. Surgical location of the electroencephalography electrodes.

in the stimulation group, and this was the same scenario for the older rats shown in Fig. 5C and D. As shown in Fig. 5B and D, all brain regions were affected; channel 8 was more active than the others when the left sciatic nerve was stimulated. Based on these phenomena, the location of channel 8 was considered to be related to the electrical stimulation of the sciatic nerve. As presented in Fig. 6, the signal was distributed relatively evenly throughout the brain at the low frequency $(<30 \mathrm{~Hz})$. At $10 \mathrm{~Hz}$, the power density of the brain region at channel 8 was relatively higher than that of other brain regions (according to the electrode distribution shown in Fig. 2A).

Data processing. The SciPy package in Python was used to generate the EEG power maps, which are shown in Fig. 7. The EEGs of non-electrical stimulation and electrical stimulation 

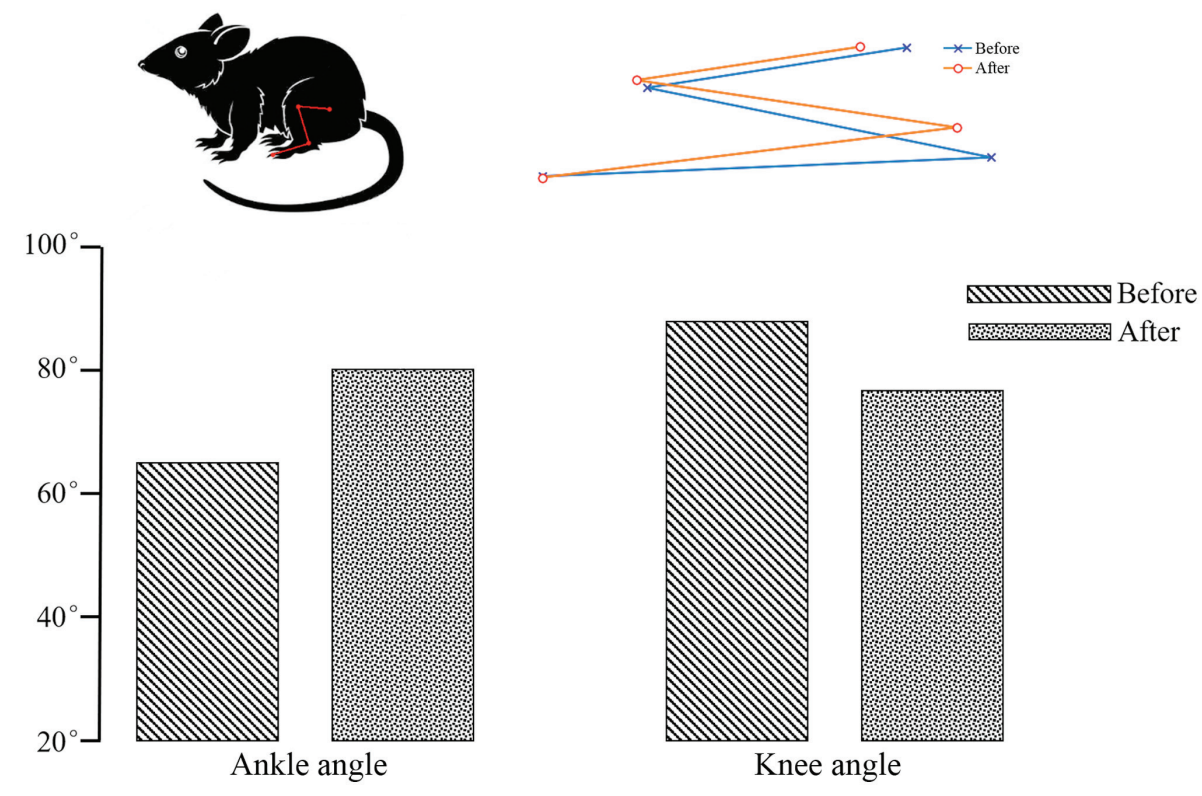

Figure 4. Angle view before and after nerve electrical stimulation. Rat leg movement when the sciatic nerve was stimulated. Blue represents the initial state; red represents the end state. The ankle angle was larger before stimulation then after; the knee angle was smaller before stimulation than after.

A

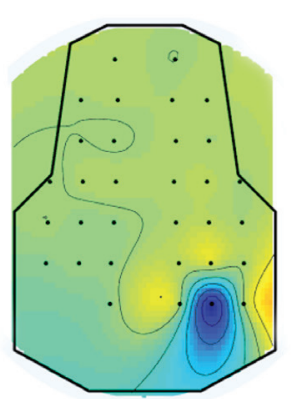

$\mathrm{C}$

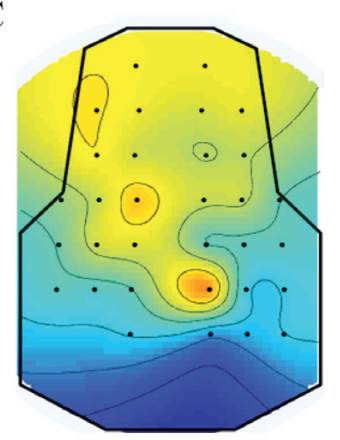

$\mathrm{B}$

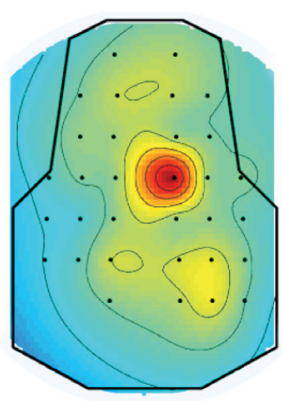

$\mathrm{D}$

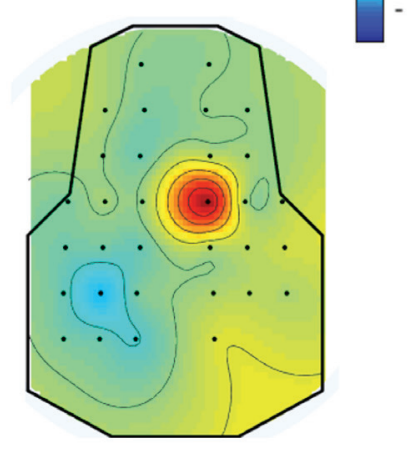

Figure 5. EEG power maps of both young and old rats under different stimulation conditions. Red color indicates strong intensity and blue indicates a weak activation status. (A) When the sciatic nerve of younger rats was not stimulated, all the brain regions were in an inactive state. The voltage intensities of all the channels were very low. (B) When the sciatic nerve of younger rats was stimulated, the most active channel was channel 8. (C) When the sciatic nerve of older rats was not stimulated, all the brain regions were in an inactive state. The amplitude of the EEG signal in the whole brain was relatively low and, in comparison, the voltage of channel 4 was relatively high. (D) When the sciatic nerve of older rats was stimulated, the most active channel was channel 8. EEG, electroencephalogram.

in younger rats are shown in Fig. 7A and B, respectively. The EEGs of non-electrical stimulation and electrical stimulation in older rats are shown in Fig. 7C and D, respectively. It was demonstrated that the voltage of channel 8 was higher than that

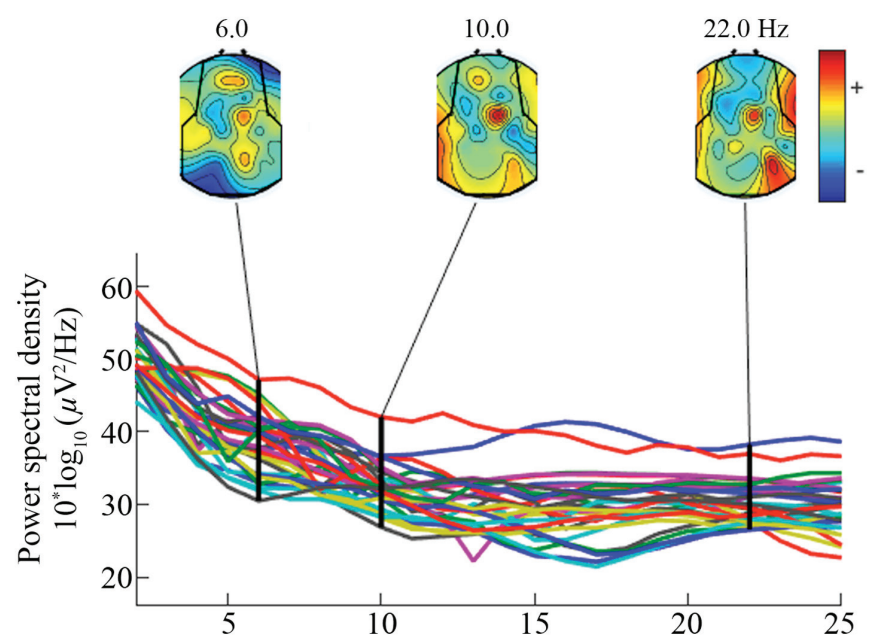

Figure 6. Frequency map of all areas of the brain. Channel 8 was activated at a frequency of $10 \mathrm{~Hz}$.

for other channels. When comparing older rats with younger rats, the same phenomenon was observed (when the left sciatic was stimulated, all the brain regions were influenced, but the right hemisphere was more strongly influenced than the left). As shown in Fig. 8A and B, wavelet analysis showed a string of low-frequency data in the period between 220 and $225 \mathrm{sec}$. At 215 and $232 \mathrm{sec}$, the rat was stimulated by the implanted electrical stimulator, and at these two points in time the wavelet analysis showed more low-frequency components than high-frequency components, indicating that electrical stimulation of the sciatic nerve can simultaneously activate the motor cortex. As shown in Fig. 9, the data of channel 8 was analyzed by computing Fast Fourier Transform. It has been established that the frequency of motion and perception is $8-16 \mathrm{~Hz}(33-35)$, and this frequency at channel 8 was higher than others in the present experiment. The results indicated that stimulation of the sciatic nerve excited the brain region under channel 8 . 
A

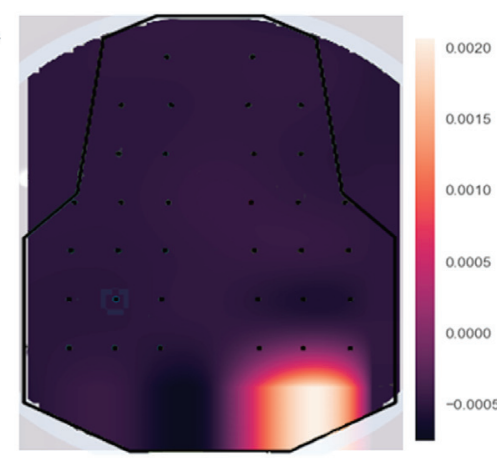

\section{$\mathrm{C}$}

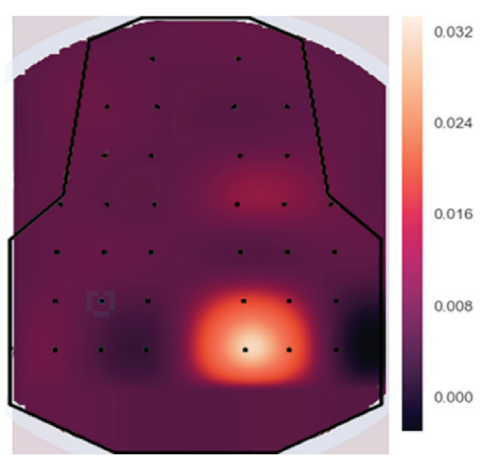

$\mathrm{B}$

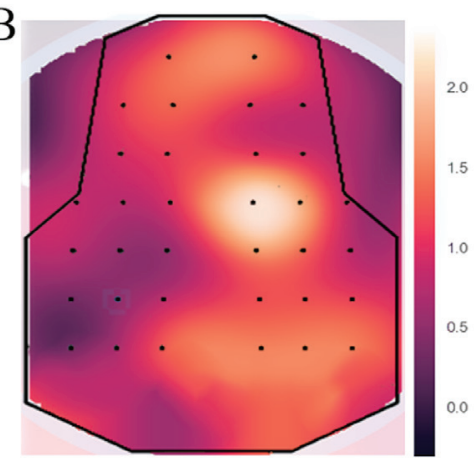

$\mathrm{D}$

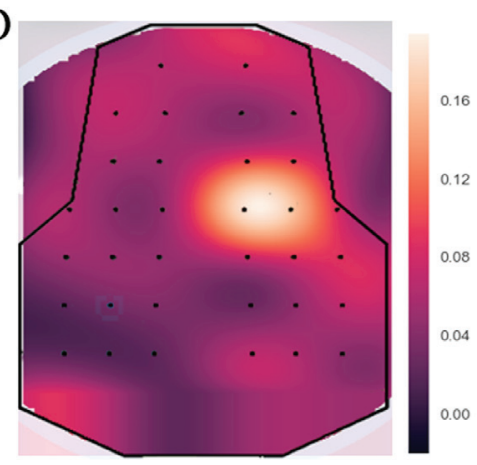

Figure 7. Power maps generated in Python. All data are expressed as the variance around $5 \mathrm{~s}$ before and after nerve electrical stimulation. (A) When the sciatic nerves of younger rats were not stimulated, the power map for all brain regions was inactive. (B) When the sciatic nerves of younger rats were stimulated, channel 8 was activated. (C) When the sciatic nerves of older rats were not stimulated, the power map for all brain regions was inactive. (D) When the sciatic nerves of older rats were stimulated, channel 8 was activated.

A

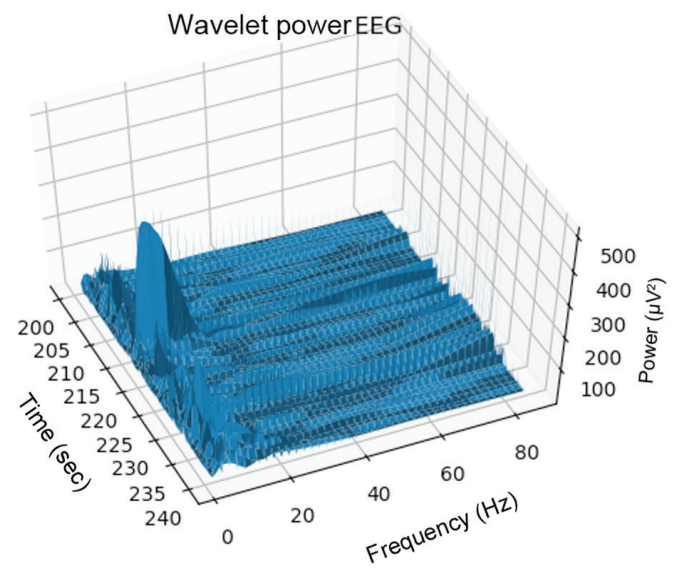

B

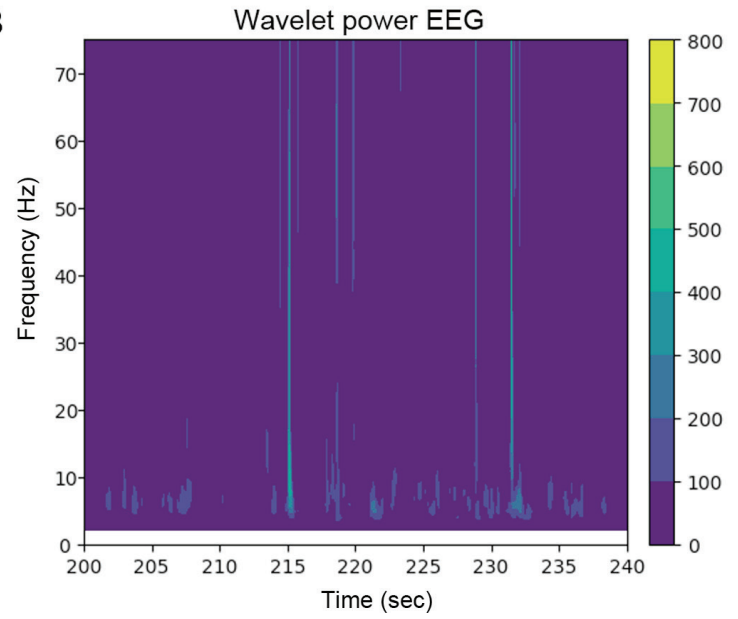

Figure 8. Wavelet transformation. Frequencies around $10 \mathrm{~Hz}$ were the main component and the voltage amplitude reached a maximum between 215 sec and $232 \mathrm{sec}$. (A) Stereogram display. (B) Heat map display. EEG, electroencephalogram.

\section{Discussion}

Stroke caused by cerebral hemorrhage is likely to become increasingly common as the population ages, thus leading to more cases of degradation of the cortex. The current treatments for improving this condition include certain medications, physical exercise and transcutaneous electrical nerve stimulation $(10,11)$, which are only capable of improving symptoms and are not curative. The usage of neuro-electrical stimulation to relieve local pain within a short period was first proposed by Wall and Sweet (36) in 1967, and it has been demonstrated that electrical stimulation affects sensory nerves $(37,38)$. In 1952, Malis et al (39) found that action potentials were generated in the motor cortex after stimulating a peripheral nerve. Based on the aforementioned studies, a new idea was proposed in the present study to treat damage to and degradation of the motor cortex by stimulating the sciatic nerve. In the present study, a self-developed fully-implanted neuro-electrical stimulator was implanted into rats, and the experimental results showed that an EEG 


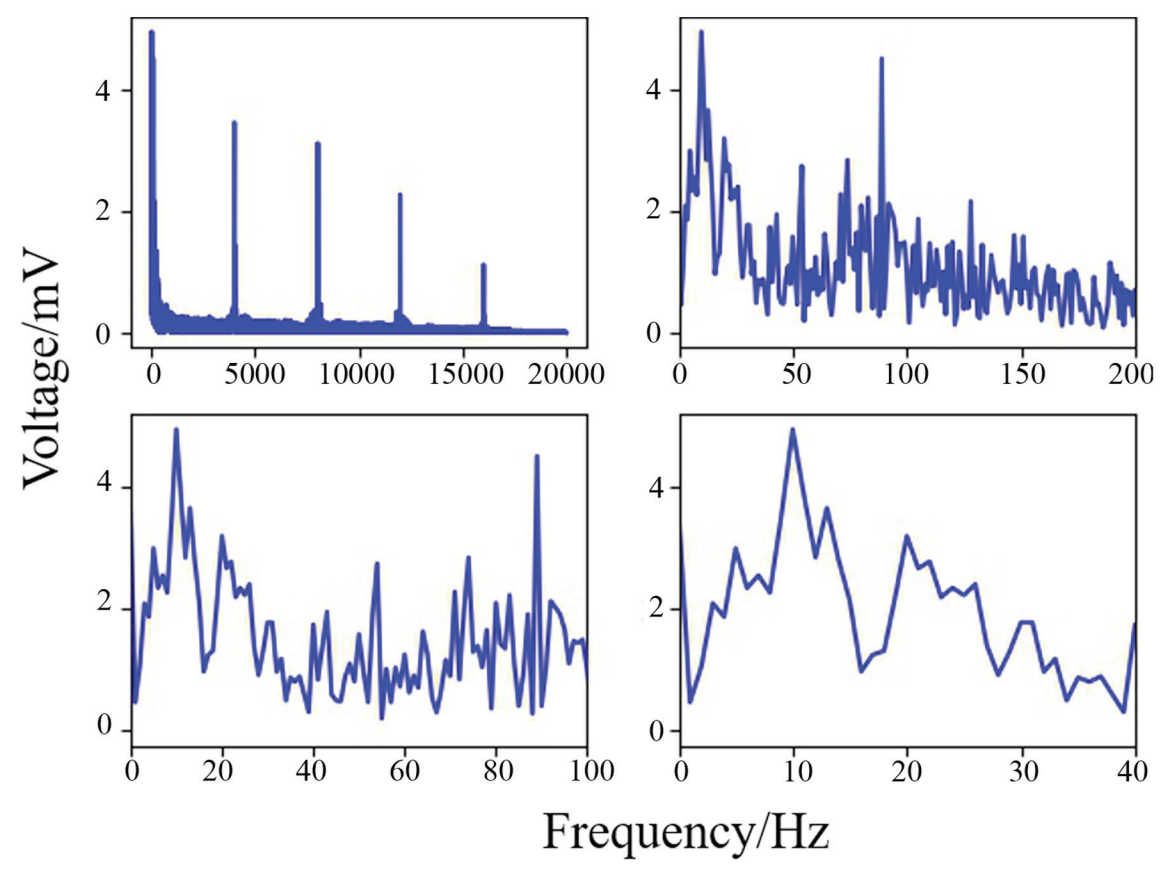

Figure 9. Spectrum of frequencies around channel 8 (the horizontal axis represents time and the vertical axis represents voltage). The voltages of frequencies $\sim 10 \mathrm{~Hz}$ were higher than at other frequencies, indicating that the voltage of the $\alpha$ band was higher than that of the $\beta, \gamma$ and $\delta$ frequency bands.

frequency band of $8-16 \mathrm{~Hz}(\alpha)$ could be evoked by electrical nerve stimulation, accompanied by leg movement, and that it had the highest amplitude of all frequency bands.

Electrodes need to be biocompatible, non-toxic and not provoke an immune response (40). Gold is utilized in EEG electrode manufacture, a material that is commonly used in surface electromyography and invasive extracellular electrodes (41). The stimulus wave in the present study was square wave, which is more efficient than other wave types (31), and the frequency of the carrier wave was $1 \mathrm{kHz}$ (avoiding damage to the nerves by continuous current). The stimulus voltage was set between $+5 \mathrm{~V}$ and $-5 \mathrm{~V}(42,43)$ (the adjustable voltage range of the self-developed neurostimulator is between $+15 \mathrm{~V}$ and $-15 \mathrm{~V}$ ). The stimulus voltage duty cycle was $50 \%$. The leg movements of the rats evoked by different stimulus voltages differed, which may be caused by slight differences in the fixation position of the electrodes. When the sciatic nerve was stimulated by electrical stimulator, the most active channel was channel 8 . Therefore, nerve electrical stimulation of the sciatic nerve can activate the motor cortex and evoke $\alpha$ wave which is related to movement $(34,35)$. During the experiment, especially for long-term nerve electrical stimulation, the use of excessive voltages was carefully avoided. In the present study, electrical stimulation experiments were performed on a daily basis for 1 month, and paralysis occurred on the surgical side of the bodies of one rat; the same response was observed in a patient after spinal nerve electrical stimulation (44), suggesting that tissue damage may occur under electrical stimulation $(45,46)$. The mode and intensity of nerve electrical stimulation require further study. The intensities of the younger rats' EEG power maps were higher than those for older rats; this may be because the number of experiments was small, or because the cortex of a younger rat is more active than that of an older rat, but this needs to be clarified in further experiments. However, the location of the brain area activated by electrical stimulation of the sciatic nerve was the same in younger and older rats. Therefore, it could be concluded that electrical stimulation of the sciatic nerve activated the corresponding motor cortex region.

In the current study, the fully-implanted nerve electrical stimulator that had been developed was able to more flexibly set a waveform cycle for nerve electrical stimulation. The wireless charging strategy avoids the disadvantages of a disposable device, the control terminal is designed to be diversified, and the size of the stimulator is small. The experiments indicated that electrical stimulation of the sciatic nerve could effectively activate specific brain regions in the rats, suggesting that, to an extent, sciatic nerve stimulation can stimulate the corresponding brain region, and also indicating, to an extent, that sciatic nerve electrical stimulation may contribute to the activation and recovery of motor cortex injury; this may provide a new method for treating stroke-induced brain damage.

\section{Acknowledgements}

The authors would like to thank Dr Luyao Chen from the School of Optical and Electronic Information, Huazhong University of Science and Technology for constructive discussions and contribution to the experimental design, the design of the stimulator and data processing for the current study.

\section{Funding}

This study was supported by Beijing Municipal Science and Technology Program (grant no.Z181100003118007), the Nation Key R\&D Program of China (grant nos. 2017YFA0701102 and 2018YFB1307300), National Natural Science Foundation of China (grant nos. 91648207 and 61673068) and the Research Fund of PLA of China (grant no. BWS17J024). 


\section{Availability of data and materials}

The datasets used and/or analyzed during the current study are available from the corresponding author on reasonable request.

\section{Authors' contributions}

$\mathrm{XL}, \mathrm{RT}, \mathrm{YL}$ and $\mathrm{JH}$ contributed to the conception and design of the study. DH and GL were responsible for the collection and processing of data. ZG and XL completed data analysis and interpretation. XL, YL and JH contributed to manuscript writing. All authors read and approved the final manuscript.

\section{Ethics approval and consent to participate}

The present study was approved by the Laboratory Animal Ethics Committee of Beijing Institute of Technology (Beijing, China).

\section{Patient consent for publication}

Not applicable.

\section{Competing interests}

The authors declare that they have no competing interests.

\section{References}

1. Gao X and Chen J: Mild traumatic brain injury results in extensive neuronal degeneration in the cerebral cortex. J Neuropathol Exp Neurol 70: 183-191, 2011.

2. Iizuka H, Sakatani $\mathrm{K}$ and Young W: Neural damage in the rat thalamus after cortical infarcts. Stroke 21: 790-794, 1990.

3. Xie H, Kane JT, Dennis MJ, Mooney RD, Bauer WR, Wang X and Wall JT: Case series evidence for changed interhemispheric relationships in cortical structure in some amputees. J Clin Neurosci 20: 523-526, 2013.

4. Salat DH, Kaye JA and Janowsky JS: Selective regional degeneration and preservation within the prefrontal cortex in healthy aging and Alzheimer's disease. Arch Neurol 58: 1403-1408, 2001.

5. Kline AE, Chen MJ, Tso-Olivas DY and Feeney DM: Methylphenidate treatment following ablation-induced hemiplegia in rat: Experience during drug action alters effects on recovery of function. Pharmacol Biochem Behav 48: 773-779, 1994.

6. Khan MA, Tariq M, Naime M and Akhtar J: Falij-E-Nisfi (Hemiplegia) cause and treatment in Unani medicine: A review. J Pharm Pharm Sci 7: 293-300, 2018.

7. Perry JC and Rosen J: Upper-limb powered exoskeleton design. IEEE/ASME Trans Mechatronics 12: 408-417, 2007.

8. Twitchell TE: The restoration of motor function following hemiplegia in man. Brain 74: 443-480, 1951.

9. Gallas S, Marie JP, Leroi AM and Verin E: Sensory transcutaneous electrical stimulation improves post-stroke dysphagic patients. Dysphagia 25: 291-297, 2010.

10. Kwong PWH, Ng GYF, Chung RCK and Ng SSM: Bilateral transcutaneous electrical nerve stimulation improves lower-limb motor function in subjects with chronic stroke: A randomized controlled trial. J Am Heart Assoc 7: pii: e007341, 2018.

11. Laddha D, Ganesh GS, Pattnaik M, Mohanty P and Mishra C: Effect of transcutaneous electrical nerve stimulation on plantar flexor muscle spasticity and walking speed in stroke patients. Physiother Res Int 21: 247-256, 2015.

12. Ghoname ESA, White PF, Ahmed HE, Hamza MA, Craig WF and Noe CE: Percutaneous electrical nerve stimulation: An alternative to TENS in the management of sciatica. Pain 83: 193-199, 1999.

13. Goldner JL, Nashold BS Jr and Hendrix PC: Peripheral nerve electrical stimulation. Clin Orthop Relat Res: 33-41, 1982.
14. Davies PM: The comprehensive treatment of patients with hemiplegia., 2000.

15. Cramer SC: Repairing the human brain after stroke: I. Mechanisms of spontaneous recovery. Ann Neurol 63: 272-287, 2008.

16. Monte-Silva K, Piscitelli D, Norouzi-Gheidari N, Batalla MAP, Archambault P and Levin MF: Electromyogram-related neuromuscular electrical stimulation for restoring wrist and hand movement in Poststroke hemiplegia: A systematic review and meta-analysis. Neurorehabil Neural Repair 33: 96-111, 2019.

17. Wilson RD, Bennett ME, Nguyen VQC, Bock WC, O'Dell MW, Watanabe TK, Amundson RH, Hoyen HA and Chae J: Fully implantable peripheral nerve stimulation for hemiplegic shoulder pain: A multi-site case series with two-year follow-up. Neuromodulation 21: 290-295, 2018.

18. Lee JH, Baker LL, Johnson RE and Tilson JK: Effectiveness of neuromuscular electrical stimulation for management of shoulder subluxation post-stroke: A systematic review with meta-analysis. Clin Rehabil 31: 1431-1444, 2017.

19. Paik YR, Park HS, Oh DH, Lee JH and Lee DH: Effect of mirror therapy and electrical stimulation on upper extremity function in stroke with hemiplegic patient: A pilot study. J Phys Ther Sci 29: 2085-2086, 2017.

20. Russo C, Souza Carneiro MI, Bolognini N and Fregni F: Safety review of transcranial direct current stimulation in stroke. Neuromodulation 20: 215-222, 2017.

21. Guiraud D, Azevedo Coste C, Benoussaad M and Fattal C: Implanted functional electrical stimulation: Case report of a paraplegic patient with complete SCI after 9 years. J Neuroeng Rehabil 11: 15, 2014.

22. Herman R, He J, D'Luzansky S, Willis W and Dilli S: Spinal cord stimulation facilitates functional walking in a chronic, incomplete spinal cord injured. Spinal Cord 40: 65-68, 2002.

23. Harkema S, Gerasimenko Y, Hodes J, Burdick J, Angeli C, Chen Y, Ferreira C, Willhite A, Rejc E, Grossman RG and Edgerton VR: Effect of Epidural stimulation of the lumbosacral spinal cord on voluntary movement, standing and assisted stepping after motor complete paraplegia: A case study susan. Lancet 377: 1938-1947, 2011.

24. Zheng ZT, Dong XL, Li YD, Gao WW, Zhou Y, Jiang RC, Yue SY, Zhou ZW and Zhang JN: Electrical stimulation improved cognitive deficits associated with traumatic brain injury in rats. Brain Behav 7: e00667, 2017.

25. Koyama S, Xia J, Leblanc BW, Gu JW and Saab CY: Sub-paresthesia spinal cord stimulation reverses thermal hyperalgesia and modulates low frequency EEG in a rat model of neuropathic pain. Sci Rep 8: 7181, 2018

26. Yip M,Jin R, Nakajima HH, Stankovic KM and Chandrakasan AP: A fully-implantable cochlear implant SoC with piezoelectric middle-ear sensor and arbitrary waveform neural stimulation. IEEE J Solid-State Circuits 50: 214-229, 2015.

27. Kim D, Yeon C, Chung E and Kim K: A non-invasive flexible multi-channel electrode for in vivo mouse EEG recording. In: Proceedings of IEEE Sensors Conference, Valencia, Spain. vol. 2-5 Novemb, pp1111-1114, 2014.

28. Zhang P, Ma X, Chen L, Zhou J, Wang C, Li W and He J: Decoder calibration with ultra small current sample set for intracortical brain-machine interface. J Neural Eng 15: 026019, 2018.

29. Zhang P, Huang J, Li W, Ma X, Yang P, Dai J and He J: Using high frequency local field potentials from multi-cortex to decode reaching and grasping movements in monkey. IEEE Trans Cogn Dev Syst: Sep, 2018 DOI: 10.1109/TCDS.2018.2869587.

30. National Institute of Health (NIH): Guide for the Care and Use of Laboratory Animals. The National Academies Press, Washington, D.C., 1996.

31. Wongsarnpigoon A and Grill WM: Energy-efficient waveform shapes for neural stimulation revealed with a genetic algorithm. J Neural Eng 7: 046009, 2010.

32. Delorme A and Makeig S: EEGLAB: An open source toolbox for analysis of single-trial EEG dynamics including independent component analysis. J Neurosci Methods 134: 9-21, 2004.

33. Klimesch W: EEG alpha and theta oscillations reflect cognitive and memory performance: A review and analysis. Brain Res Rev 29: 169-195, 1999.

34. Chatrian GE, Petersen MC and Lazarte JA: The blocking of the rolandic wicket rhythm and some central changes related to movement. Electroencephalogr Clin Neurophysiol 11: 497-510, 1959. 
35. Babiloni C, Carducci F, Cincotti F, Rossini PM, Neuper C, Pfurtscheller G and Babiloni F: Human movement-related potentials vs desynchronization of EEG alpha rhythm: A high-resolution EEG study related to movement. Neuroimage 10: 658-665, 1999.

36. Wall PD and Sweet WH: Temporary abolition of pain in man. Science 155: 108-109, 1967

37. Slavin KV: Peripheral nerve stimulation for neuropathic pain. US Neurol 7: 144, 2015

38. Levine AB, Steven DA, Parrent AG and MacDougall KW: Successful long-term nerve root stimulation for chronic neuropathic pain: A real world, single center Canadian experience. Pain Physician 20: 95-106, 2017.

39. Malis LI, Pribram KH and Kruger L: Action potentials in 'Motor' cortex evoked by peripheral nerve stimulation. Methods 16 : 161-167, 1953.

40. Merrill DR, Bikson M and Jefferys JG: Electrical stimulation of excitable tissue: Design of efficacious and safe protocols. J Neurosci Methods 141: 171-198, 2005.

41. Dong W, Zhu C, Hu W, Xiao L and Huang Y: Stretchable human-machine interface based on skin-conformal sEMG electrodes with self-similar geometry. J Semicond 39: 014001, 2018.
42. O'Suilleabhain PE, Frawley W, Giller C and Dewey RB Jr: Tremor response to polarity, voltage, pulsewidth and frequency of thalamic stimulation. Neurology 60: 786-790, 2003.

43. Mooziraji FB and Shoaei O: A high power efficient multi-waveform current stimulator used in implantable neural stimulation. Analog Integr Circuits Signal Process 86: 459-469, 2016.

44. Shealy CN, Mortimer JT and Reswick JB: Electrical inhibition of pain by stimulation of the dorsal columns: Preliminary clinical report. Anesth Analg 46: 489-491, 1967.

45. Cogan SF, Ludwig KA, Welle CG and Takmakov P: Tissue damage thresholds during therapeutic electrical stimulation. J Neural Eng 13: 21001, 2016.

46. Levy RM: Device complication and failure management in neuromodulation. Neuromodulation 16: 495-502, 2013.

cc) (i) (3) This work is licensed under a Creative Commons Attribution 4.0 International (CC BY-NC 4.0) License 